\title{
MUSINGS
}

\section{A personal perspective on returning secondary results of clinical genome sequencing}

\author{
David Dimmock*
}

\section{Clinical tests and the duty of care}

Although much has been written about the return of research results from clinical trials, and these can inform much of the discussion around results for clinical patients, the return of results from clinical testing is different. It is informed by a different kind of relationship, one in which there is a duty of care on the clinician to the patient [1]. There is a different framework for liability in terms of not acting or disclosing results that a reasonable person would want to know. Although the legal opinions on disclosure are beyond the scope of this article, many laboratory professionals consider that there is a duty of care to return off-target results by a laboratory if these are 'actionable' (that is, if they would generally lead to a change in care), even when such a test was not ordered. An example of such an actionable off-target result would be if the results from an automated machine showed a critical platelet count when a test for hemoglobin has been ordered [2]. Nonetheless, there are no clear statutory or professional recommendations. In several US states there are specific laws that may be construed as stating that DNA test results are considered the property of the tested individual [3]. Therefore, there is the potential for a moral or legal obligation to return results when such information would lead to a change in current clinical management. However, it is unclear what obligations there should be on laboratories that evaluate genomes to search for such secondary findings. In addition, it is ambiguous what should be considered a result that 'would change clinical management' - for example, whether a result needs to lead to a change in treatment, or whether making a diagnosis is sufficient to be considered a change in management. Furthermore, it is unclear who should decide when something reaches this standard. This decision-making becomes considerably more difficult in the situation of individuals for whom a proxy decision maker is empowered, such as

*Correspondence: ddimmock@mcw.edu

Division of Genetics, Department of Pediatrics and Human and Molecular Genetics Center, Medical College of Wisconsin, Milwaukee, WI 53221, USA adults considered incompetent through illness of mental deficiency, and minors. Here, I provide a clinician's perspective on the issues of secondary results raised by genome-wide sequencing and consider strategies that have been implemented to address these issues.

\section{Secondary findings in clinical genomics}

The issues of off-target genomic data are not new to the clinical genetics community. Indeed, genome-wide copy number testing of minors has raised ethical issues with regard to variants of uncertain significance and the identification of adult-onset disease-causing variants. Consequently, centers have had to establish an approach to the consent and management of such results. Examples of potentially actionable off-target results at our center have included $Y$ chromosome deletions that may lead to infertility, HNF4A deletions that may predispose to diabetes, deletions of genes that would predispose to cancer for which screening is recommended at varying ages (such as RB1, MSH6, and SMAD4), and deletions that alter therapeutic drug management (such as a deletion of PMP22 predisposing to vincristine toxicity in a patient with pediatric leukemia).

The entry of whole exome or whole genome sequencing (WGS) over the past 2 years into limited clinical practice for children with rare disorders has led to a widening of the availability of genome-scale testing beyond copy number arrays [4]. With the consequent higher frequency of detection of genomic variants, establishing a plan to deal with secondary results becomes more important.

With mutations in over 2,000 genes currently causally associated with defects in human health, it is unrealistic to provide a definitive list of all variants that should or should not be considered for return. As a result of allelic heterogeneity in many disease-causing genes - for example, $L M N A$, which has more than ten clinical phenotypes associated with it that may be childhood or adult onset it is also inappropriate to stratify genetic changes by the gene involved. Therefore, it is necessary to stratify each specific genomic change by the predicted effects on the health of the individual and the confidence that it will lead to the health outcome expected $[5,6]$. 
Table 1. Examples of categories of health effects stratified by age of onset

\begin{tabular}{lll}
\hline & Recognized therapeutic or preventive intervention & Untreatable \\
\hline Childhood onset & Biotinidase deficiency, pharmacogenomic variants & Tay Sachs disease \\
Adult onset & Breast cancer predisposition caused by mutations in BRCA1 & Parkinson's disease caused by mutations in PARK1 \\
\hline
\end{tabular}

These health effects can be stratified by age of onset into childhood-onset and adult-onset effects, and we have subdivided these into those in which there is a recognized preventive or therapeutic intervention and those in which there is not [5] (Table 1). Although most ethicists agree that utility is required before returning test results, there is considerable controversy about the precise threshold of utility that is required before a result can or should be returned in the research setting [7], and similar conversations can be expected in the clinical sphere. Some authorities have taken the position that proven clinical utility to the individual having their genome sequenced is necessary (that is, that some recognized preventive or therapeutic intervention must exist) before returning results $[6,8]$. Others assert that utility to the wider family (for example, the possibility that the information could be used for family planning) is relevant [9]. What has been less clear is the approach to take for variants that may affect risks of developing disease, or those that may have more personal rather than clinical utility (such as information on ethnic origin). Assessments of clinical utility, especially for secondary results, may also depend on cultural and philosophical beliefs of the patient and, if relevant, their care-givers. Furthermore, it is unclear what level of proof should be required to assert that a variant will have the expected clinical effect.

Given the lack of consensus in this area, there is a need for a discussion about what types of information can, should, or should not be offered to patients in a range of clinical contexts, and about who should determine what results should be returned.

\section{A way forward}

When we embarked upon a clinical WGS program for children in late 2009, we consulted with multiple ethicists, both internally and at external institutions, to develop a pragmatic framework in which to return results [5]. After education and counseling, parents elect to receive information based on their beliefs of utility for variants with an established disease association.

Clinical utility for the tested individual is clearly apparent for childhood-onset disorders and pharmacogenomic disorders in children, and it is our institutional policy to mandate return of treatable childhood-onset disorders. We are considering whether mandatory return, to the ordering physician or directly into the electronic medical record, should be implemented for pharmacogenomic variants with the highest level of evidence, that is, Clinical Pharmacogenetics Implementation Consortium of the Pharmacogenomics Research Network (CPIC) Level 1 variants.

The case for clinical utility in returning adult-onset treatable disorders is relatively uncomplicated. Specifically, detection of, for instance, a known pathogenic mutation in the breast cancer gene BRCA1 in a child has implications for the testing and health surveillance of the child's parent who carries the mutation. In this instance the benefits to the child in improving parental health outcomes can be seen to justify the child's loss of autonomy in not being able to ultimately choose not to know their own status.

Perhaps less intuitive, but commonly raised by families with whom we have discussed WGS, is the utility of knowledge about 'untreatable' disorders in future family decision-making. Many parents express a desire to know whether their child harbors such mutations, saying that it will change their current decisions - for instance, by not having further children or considering pre-implantation genetic diagnosis. The ethical weight of such parental choice over the child's autonomy is difficult to gauge. More significant is the ethical question of who has the right to choose which to favor - the parent's ability to manage their decisions or the child's autonomy. In general in Western societies, it is considered that parents are in the best position to make such choices over their child's healthcare and lifestyle decisions.

At our institution, after significant internal and external discussion with ethicists, geneticists, legal counsel and several groups of parents, we have come to the conclusion that parents themselves are in the best position to choose what information should be returned to them. Therefore, in the context of the categorical model of choice, we will return at the parent's request data on affected status for treatable and untreatable disease where we have definitive pathogenic mutations.

Clinical sequencing will identify variants in the genome that are published as conferring increased or reduced risk of developing disease, such as single nucleotide polymorphisms that are associated with increased risk of developing diabetes mellitus or heart disease. The strength of such associations must be considered, taking into account any prior risk of developing disease and the extent to which such risk is changed by the family history [10]. Because of this uncertainty, we have chosen not to return such variants. 
Over the subsequent 2 years of our clinical WGS program for children it has been interesting to note the wide variety of results-return options selected by parents of children undergoing clinical WGS at our institution. More than half of parents who have proceeded with WGS have elected to learn about adult-onset information. Some families have elected to receive information about adult-onset treatable conditions but not untreatable childhood conditions. This range of choices selected by parents from one center suggests that we should not pursue a policy of 'one size fits all' for data return.

The face-to-face education and counseling required for our current approach are not feasible for mass market delivery. However, our center and many others are receiving positive early opinions from consumers of computeror web-based information delivery. Further research will be required to ensure that such tools live up to early promise. However, it appears that they will enable consumers to continue to exercise nuanced consent for secondary results return.

Consequently, irrespective of information and consent approaches, we will be able to continue to offer a variety of data-return options.

\section{Acknowledgements}

While the opinions presented in this article are mine, I wish to acknowledge the Children's Hospital of Wisconsin WGS review group for many stimulating conversions, especially Rodney Willoughby, Steve Leuthner, Regan Veith, Michael Gutzeit, Tom May and David Bick. I am grateful to the Genetic Counseling staff and Clinical Laboratory Staff at CHW/Medical College of Wisconsin, specifically David Bick, Regan Veith, Daniel Helbling, Jamie WendtAndrae, Michael Tschannen, Howard Jacob and Elizabeth Worthey. In addition, I am grateful to the many volunteers and financial donors who have made the clinical program a reality.

\section{Competing interests}

The author declares that he has no competing interests.

\section{Abbreviations}

CPIC, Clinical Pharmacogenetics Implementation Consortium of the

Pharmacogenomics Research Network; WGS, whole-genome sequencing.

Published: 26 June 2012
References

1. Clayton EW, McGuire AL: The legal risks of returning results of genomics research. Genet Med 2012, 14:473-477.

2. Karon BS: Handling values that are not ordered [http://www.mlo-online com/features/201110/tips-from-the-clinical-experts/answering-yourquestions.aspx]

3. Wagner JK, Vorhaus D: On Genetic Rights and States: a Look at South Dakota and Around the U.S. [http://www.genomicslawreport.com/index. php/2012/03/20/

on-genetic-rights-and-states-a-look-at-south-dakota-and-around-the-u-s/]

4. Bick D, Dimmock D: Whole exome and whole genome sequencing. Curr Opin Pediatr 2011, 23:594-600.

5. Mayer AN, Dimmock DP, Arca MJ, Bick DP, Verbsky JW, Worthey EA, Jacob HJ, Margolis DA: A timely arrival for genomic medicine. Genet Med 2011, 13:195-196.

6. Berg JS, Khoury MJ, Evans JP: Deploying whole genome sequencing in clinical practice and public health: meeting the challenge one bin at a time. Genet Med 2011, 13:499-504.

7. Evans JP, Rothschild BB: Return of results: not that complicated? Genet Med 2012, 14:358-360.

8. Green RC, Berg JS, Berry GT, Biesecker LG, Dimmock DP, Evans JP, Grody WW, Hegde MR, Kalia S, Korf BR, Krantz I, McGuire AL, Miller DT, Murray MF, Nussbaum RL, Plon SE, Rehm HL, Jacob HJ: Exploring concordance and discordance for return of incidental findings from clinical sequencing. Genet Med 2012, 14:405-410

9. Richards CS, Bale S, Bellissimo DB, Das S, Grody WW, Hegde MR, Lyon E, Ward BE; Molecular Subcommittee of the ACMG Laboratory Quality Assurance Committee: ACMG recommendations for standards for interpretation and reporting of sequence variations: Revisions 2007. Genet Med 2008, 10:294-300.

10. Heald B, Edelman E, Eng C: Prospective comparison of family medical history with personal genome screening for risk assessment of common cancers. Eur J Hum Genet 2012, 20:547-551.

doi:10.1186/gm353

Cite this article as: Dimmock D: A personal perspective on returning secondary results of clinical genome sequencing. Genome Medicine 2012, 4:54 